\title{
Exercise and air quality: 10 top tips
}

1

\section{Consider your location and route}

When exercising in a town or city, make use of parks, public spaces and trails with low emission zones as much as possible. A growing amount of research is showing that greenspaces contribute to our health and wellbeing.

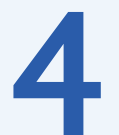
Avoid busy roads
with high buildings

Air pollution tends to get trapped within roads with tall buildings on either side, so this type of road usually has poor air quality and should be avoided for exercise. Traffic lights are another pollution hotspot to avoid, as when vehicles move away from traffic lights they give off more emissions.

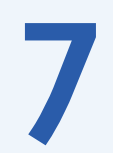

\section{Avoid exercising during rush hours}

Try to avoid being physically active outdoors during rush hour in busy traffic areas or other times when lots of cars will be on the roads. Choose an alternative, quieter route or another time to be active.

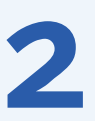

\author{
Keep a healthy \\ distance from the \\ road
}

If you are cycling, jogging or walking outdoors, it is best to avoid doing this close to roads - particularly busy ones - if possible. Studies have shown that air pollution levels are higher on busier roads, but that the amount of pollution drops in line with how far away you are from a road. For example, you will be exposed to lower levels of air pollution just $1-2 \mathrm{~m}$ away from the main flow of traffic. In addition, you may want to consider using a parallel road that is quieter and safer.

\section{- Check the air quality index of the day Actual air pollution levels depend on the type of} pollutant, the location and local weather. Many government agencies have monitoring stations that continuously measure and report levels of different air pollutants. Some also give forecasts, which you could use to decide when would be better to do your outdoor exercise. Try to find a service that is specific for your location.

You can find Europe-wide air quality reports at: http://atmosphere. copernicus.eu/services/air-qualityatmospheric-composition

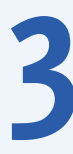

\section{Move around vehicles}

When cycling, jogging or walking behind cars, scooters, motorcycles, lorries and other vehicles, you will breathe in very high levels of pollutants that can be harmful to your lungs. If it is safe to do so, move around these vehicles to try to reduce your exposure and keep your distance.

\section{Check the weather forecast}

Air pollution tends to be at its highest on hot, sunny days, while the air tends to be cleaner after rainy or windy weather. If you have a pollen allergy, you may have more issues on days where pollen levels are high as pollen can interact with pollution. Check your local air quality forecast for more information.

\section{Choose healthy
travel options \\ ( Driving a car to get around contributes to the} air pollution problem. Why not consider using public transport, or 'taking the active option' and walking or cycling? These options will help you to reach your daily physical activity goals, while contributing to a cleaner environment.

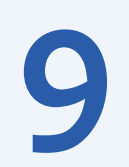

\section{Avoid exposure to indoor air pollution and second-hand smoke}

If you exercise at a gym, at home or in another indoor space, you could come into contact with indoor air pollution and/or second-hand smoke, so it is worth considering potential pollutants in this environment and how you can protect yourself from them. Vacuuming, and using cleaning products and air fresheners may reduce air quality, so try to avoid exercise immediately after cleaning.

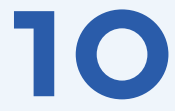

\section{Be active!}

Do not be afraid to be active and exercise - environmental and lung health experts agree that the health risks associated with breathing in air pollution while exercising are less significant than those of an inactive lifestyle. 
Physical activity covers any type of movement of the muscles in the body which uses up energy. It includes activities you may do as part of your daily routine, like gardening, cleaning or walking to the shops. Exercise is a form of physical activity that is planned, structured, repetitive, and aims to improve or maintain physical fitness.

Both gentle and more strenuous physical activity can benefit your health and improve your quality of life. Being active is also important for lung health, both for the general population and people living with long-term lung conditions.

How much and what type of exercise should I do?

There is a level of exercise or physical activity that is suitable for everyone, whether you have a lung condition or not. This could be walking, cycling, swimming, playing team sports, doing strength training or doing day-to-day activities, such as gardening or cleaning, as long as it is enough to make you moderately breathless.

If you are unsure about the type or amount of activity you should do, you could ask your healthcare professional for advice.

\section{Dhy should I consider the quality of the air I exercise in?}

When you are physically active, you breathe more often and take more air into your lungs than when you are inactive. If the air quality is poor, you may breathe in a larger amount of harmful pollutants.

In addition, while exercising, you are more likely to breathe through your mouth rather than your nose. Unlike the nose, the mouth is unable to filter out certain large pollutants in the air and stop them from entering the lungs. Therefore, breathing through the mouth can lead to more pollutants entering the airways. During exercise, smaller inhaled particles can get deeper into the lungs.

The more pollutants that you breathe into your lungs, the more likely you are to experience their negative health effects.

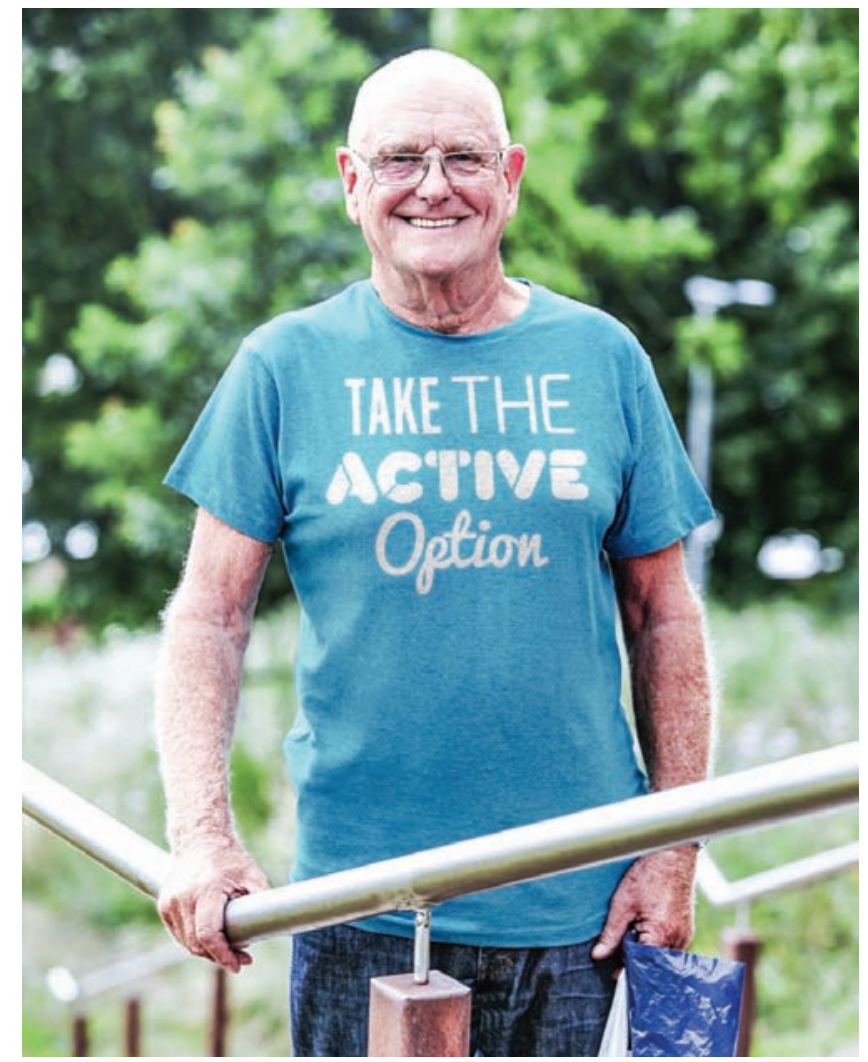


Air pollution is a mixture of particulate matter and gases. Several of these pollutants, alone or mixed together, can damage your health. It is very important to consider the impact of the air you exercise in, as when you are physically active you breathe in more air and particles can penetrate deeper in the lungs.

\section{Dow could air pollution affect my lungs?}

Exposure to air pollution can negatively affect everyone. It can be particularly harmful to people with lung conditions, such as asthma and chronic obstructive pulmonary disease (COPD), older people, children and babies.

If you have a lung condition, air pollution can cause flare-ups of your symptoms, including asthma attacks, COPD exacerbations, difficulty breathing, wheezing, coughing and irritation.

Studies have shown that, in the long term, regular exposure to air pollution is linked to the development of certain lung conditions among healthy people. Air pollution has also been associated with shortening life expectancy.

\section{What types of air pollution should I look out for when I exercise?}

There are two main types of air pollution: indoor air pollution and outdoor air pollution.

\section{Outdoor air pollution}

Outdoor air pollution includes pollutants such as particulate matter and gases such as ozone, nitrogen dioxide and sulphur dioxide. It comes from a range of sources, such as traffic and industry.

While you exercise outdoors, it is worth considering that the following areas could be more likely to be polluted:

- Major, built-up cities

- Busy roads and motorways

- Industrial areas

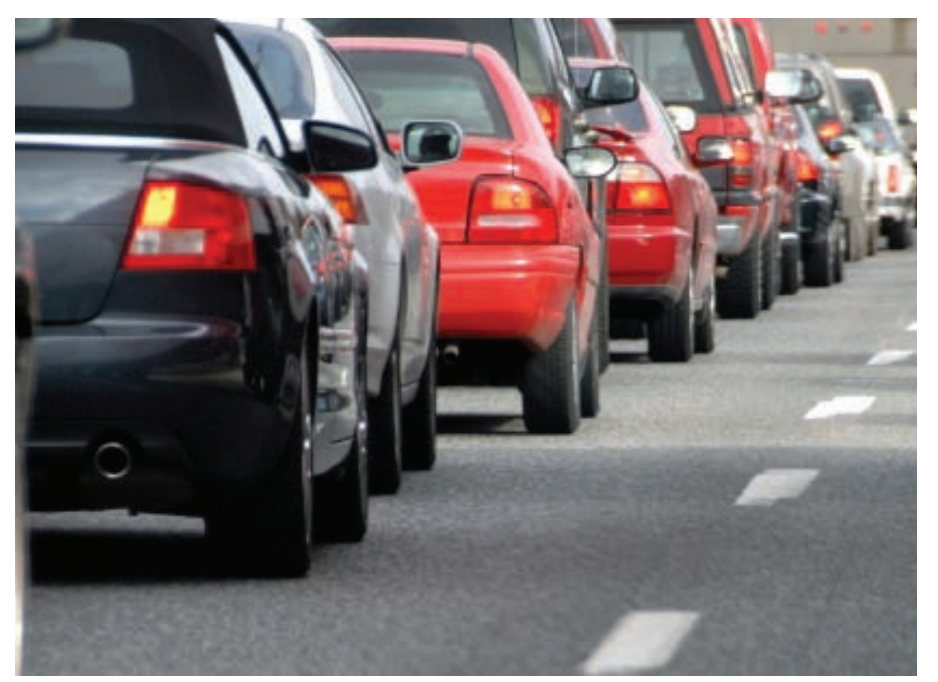

\section{Indoor air pollution}

Indoor air pollution can come from many sources, including open fires and heaters, building materials and furniture, cleaning products, cooling systems, second-hand smoke, and pollution that comes in from outdoors.

If your home, gym, swimming pool or other indoor exercise space has poor indoor air quality, your lungs could be affected by air pollution. 
"When I am walking around in Brussels, I often put a scarf across my face, as there is a lot of heavy diesel pollution here, which I really feel. It makes me feel quite anxious as I am aware that I am in pollution and that it can affect my lungs yet there is nothing I can do about it. Physically, it is not an immediate thing; I will notice at the end of the day that my lungs feel very heavy and irritated. I also cough a lot and bring up mucus.

"I live by the saying, "if you don't use it, you lose it". It is important to move and to keep your muscles going. I cycle on an indoor exercise bike, as I am unable to cycle on the roads due to all of the fumes from car exhausts. I swim, but only in outdoor swimming pools, as I cannot be in enclosed pools due to the chemicals in the air."

Caroline Gillissen, Belgium, who has alpha-1-antitrypsin deficiency

\section{Further reading}

- Visit the European Lung Foundation website for more information on air pollution, physical activity and specific lung conditions: www.europeanlung.org

- The ELF factsheet, 'Your lungs and exercise', contains more information on how exercise affects the lungs, how breathing is influenced by activity and the benefits of exercise for people with and without a lung condition.

- The ELF factsheets, 'Outdoor air pollution and the lungs' and 'Indoor air pollution and the lungs', explain more about the two main types of air pollution, their causes and how you can protect your lungs from them.

- Find out more information on the Healthy Lungs for Life campaign, including information on the importance of clean air and being physically active : www.healthylungsforlife.org

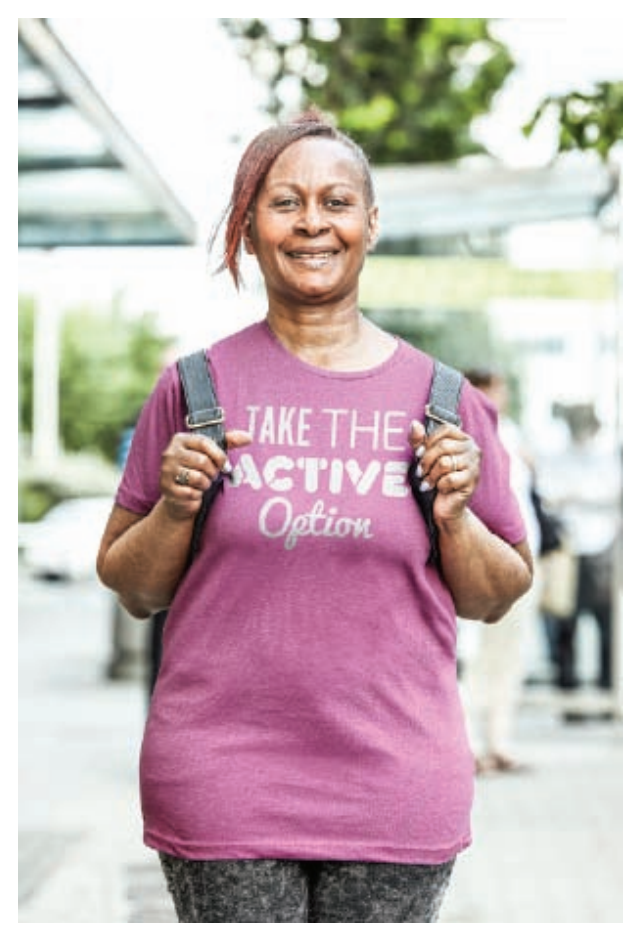

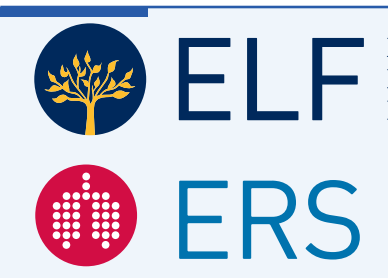

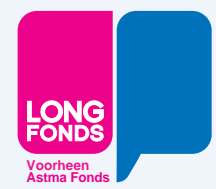

EUROPEAN LUNG FOUNDATION

EUROPEAN RESPIRATORY SOCIETY
This material was compiled with the help of Professor Benoit Nemery, Dr Frits Franssen, Professor Dr Patrick De Boever and Caroline Gillissen.

ELF was founded by the European Respiratory Society (ERS), with the aim of bringing together patients and the public with respiratory professionals to positively influence lung health. ELF is dedicated to lung health throughout Europe, and draws together the leading European medical experts to provide patient information and raise public awareness about respiratory disease.

The Lung Foundation Netherlands (Longfonds) fights for healthy lungs and against incurable lung diseases. The Netherlands counts over a million people suffering from a lung disease, including asthma and COPD. Longfonds stands up for those people, because lungs are a matter of life and death. 